\section{Converting Incidence and Prevalence Data: An Update to the Rule}

To the Editor-In developing an automated surveillance tool for healthcare-associated (HCA) urinary tract infections (UTIs), we came across a familiar issue, which was a lack of reliable, comparable data with which to test the accuracy of the tool. The automated surveillance that we developed presented an incidence, whereas the only data we had locally available regarding rates of HCA UTI were from a point prevalence survey. In this letter, we propose an amendment to a previously published formula for converting incidence and prevalence and discuss the issues involved in attempting to do so. ${ }^{1}$

The point prevalence survey was conducted at Imperial College Healthcare National Health Service Trust (London, UK) in a single, large, tertiary referral hospital as part of the European Centre for Disease Control (ECDC) Europe-wide prevalence survey for HCA infections, invasive device use, and antibiotic prescribing. ${ }^{2}$ The survey was conducted using the standard published guidelines in September 2011 and demonstrated an HCA UTI prevalence of $3.2 \%$.

The automated surveillance tool for HCA UTI was based on relevant, routinely available, electronic hospital data. Cases of HCA UTI were defined using a positive microbiology culture in line with the ECDC definitions, ${ }^{3}$ combined with information on duration of hospitalization, readmission, and presence of a urinary catheter. This covered a period of 6 months from September 2011 through March 2012, during which time there was an incidence of 1.9 UTIs per 100 admissions.

We used the method presented by Rhame and Sudderth for converting prevalence to incidence and vice versa. The formula parameters are defined in Table 1, and the values we used were derived from the point prevalence survey and the local administrative hospital data from the routine patient administration system data. The date of HCA UTI onset was recorded in the point prevalence survey, so this was used to determine the average duration from admission to HCA UTI diagnosis. The formulas are $I=P \times[\mathrm{LA} /(\mathrm{LN}-\mathrm{INT})]$ and $P=I \times[(\mathrm{LN}-\mathrm{INT}) / \mathrm{LA}]$ in which $I$ is incidence, $P$ is prevalence, LA is mean length of stay for all patients, $\mathrm{LN}$ is mean length of stay for patients with an HCA UTI, and INT is the interval between admission and infection onset.

The results of converting the prevalence and incidences are presented in Table 2, using various permutations of the formula. Converting the prevalence from the point prevalence survey based on these parameters gave an incidence of 0.56 infections per 100 admissions. This was dramatically less than expected on the basis of the results of the laboratory-based automated surveillance. The established formula estimates the duration of infection with the term (LN-INT), and based on the data we had available locally, this gave an average length of an HCA UTI as 20 days. The local antibiotic policy for an HCA UTI recommends a 7-day course of antibiotics, and if the infection is complicated, this increases to 14 days; this suggests that durations of HCA UTI are generally much shorter than the formula estimate of 20 days.

We performed the conversions using both the complicated and uncomplicated durations of antibiotic prescription to reflect the duration of infection, instead of the defined formula parameter, the justification being that, once the treatment for an infection has finished, it can be asserted that the infection has cleared. This gave incidences of 2.29 infections per 100 admissions for the short treatment course and an incidence of 1.14 using the longer treatment course. The incidence of 1.9 infections per 100 admissions lies between the values derived from using the 2 antibiotic treatment durations.

We acknowledge that the incidence value from the laboratory-based system is unlikely be a true reflection of the infections occurring in the hospital, because it is based on laboratory-confirmed cases only. The point prevalence survey

TABLE 1. Parameters Used for the Conversion of Healthcare-Associated (HCA) Urinary Tract Infection (UTI) Incidence and Prevalence

\begin{tabular}{lcc}
\hline Parameter definition & Formula parameter & Value \\
\hline Prevalence & $P$ & $3.2 \%^{\mathrm{a}}$ \\
Incidence & $I$ & $1.9^{\mathrm{b}}$ \\
Mean length of stay for patients with HCA UTI & LN & 31 days \\
Mean interval between admission and HCA UTI onset & INT & 11 days \\
Mean length of stay for all patients & LA & 5 days \\
Minimum local antibiotic course duration for HCA UTI & $\ldots$ & 7 days \\
Maximum local antibiotic course duration for HCA UTI & $\ldots$ & 14 days
\end{tabular}

\footnotetext{
a Derived from a local point prevalence survey.

b Derived from an automated surveillance tool based on laboratory-confirmed cases and local administrative hospital data.
} 
TABLE 2. Urinary Tract Infection Incidence and Prevalence

\begin{tabular}{lcc}
\hline Formula version & $\begin{array}{c}\text { Incidence } \\
\text { (measured: 1.9) }\end{array}$ & $\begin{array}{c}\text { Prevalence } \\
\text { (measured: 3.2) }\end{array}$ \\
\hline Published $^{\mathrm{a}}$ & 0.56 & 7.60 \\
Adjusted 1 $^{\mathrm{b}}$ & 2.29 & 2.66 \\
Adjusted 2 $^{\mathrm{c}}$ & 1.14 & 5.32 \\
\hline
\end{tabular}

a The formula published by Rhame and Sudderth. ${ }^{1}$

b Using the minimum antibiotic treatment duration of

7 days as the length of infection.

${ }^{c}$ Using the maximum antibiotic treatment duration of 14 days as the length of infection.

allowed for cases of HCA UTI to be diagnosed on the basis of clinical symptoms only, meaning that we are not necessarily comparing like with like. However, in this scenario, the assumption is that the incidence would be underestimated in a laboratory system. Using the formula as it is presented by Rhame and Sudderth ${ }^{1}$ for converting the prevalence to incidence instead shows the laboratory-based incidence to be an overestimation.

Earlier studies have commented on the questionable accuracy of this formula for converting incidence and prevalence data. ${ }^{4-6}$ There are important arguments for the poor performance of the conversion. First, the types of data being compared represent different diagnostic criteria. Second, reflecting the argument put forward by Hoare et al, ${ }^{5}$ patients who contract HCA UTI are generally older and have comorbidities. Therefore, using their time in the hospital from diagnosis to discharge to reflect duration of infection is unreasonable.

Using antibiotic treatment as a proxy for infection duration greatly improved the comparability of the prevalence and incidence values, and we would recommend amending this formula to the following: $I=P \times(\mathrm{LA} / \Delta \mathrm{Rx})$ and $P=I \times$ $(\Delta \mathrm{Rx} / \mathrm{LA})$, in which $I$ is incidence, $P$ is prevalence, $\mathrm{LA}$ is mean length of stay for all patients, and $\Delta \mathrm{Rx}$ is the duration of infection treatment.

\section{Carina King, PhD, MSc; ${ }^{1,2}$ Paul Aylin, MBChB, FFPHM; $;$, Alison Holmes, MD, $\mathbf{M P H}^{2,4}$}

Affiliations: 1. Institute of Global Health, University College London, United Kingdom; 2. The National Centre for Infection Prevention and Management, Imperial College, London, United Kingdom; 3. Dr Foster Unit at Imperial, School of Public Health, Imperial College London, London, United Kingdom; 4. Infection Prevention and Control, Imperial College Healthcare National Health Service Trust, London, United Kingdom.

Address correspondence to Carina King, Institute for Global Health, 30 Guilford Street, London, WC1 1NE, United Kingdom (c.king@ucl.ac.uk). Infect Control Hosp Epidemiol 2014;35(11):1432-1433

(C) 2014 by The Society for Healthcare Epidemiology of America. All rights reserved. 0899-823X/2014/3511-0020\$15.00. DOI: 10.1086/678435

\section{REFERENCES}

1. Rhame FS, Sudderth WD. Incidence and prevalence as used in the analysis of the occurrence of nosocomial infections. Am J Epidemiol 1981;113(1):1-11.

2. European Centre for Disease Prevention and Control (ECDC). Point prevalence survey of healthcare-associated infections (HAI) and antimicrobial use in European acute care hospitals-protocol version 4.3. Stockholm: ECDC, 2012.

3. Health Protection Agency. English national point prevalence survey of healthcare-associated infections and antimicrobial use, 2011: preliminary data. London: Health Protection Agency, 2012.

4. Gastmeier PMD, Bräuer H, Sohr DP, et al. Converting incidence and prevalence data of nosocomial infections: results from eight hospitals. Infect Control Hosp Epidemiol 2001;22(1):31-34.

5. Haore HG, Muller A, Daniel Talon P, Xavier Bertrand P. Estimation of the cumulative incidence of hospital-acquired bacteremia from prevalence data: a formula. Infect Control Hosp Epidemiol 2005;26(4):415-417.

6. Berthelot P, Garnier F, Fascia P, et al. Conversion of prevalence survey data on nosocomial infections to incidence estimates: a simplified tool for surveillance? Infect Control Hosp Epidemiol 2007;28:633-636.

\section{Redefining the National Healthcare Safety Network's Definition of Catheter-Associated Urinary Tract Infections: The Hazard of Including Candida Species}

\section{ACKNOWLEDGMENTS}

Financial support. The UK Clinical Research Collaboration funds the National Centre for Infection Prevention and Management at Imperial College London, which is also supported by the UK National Institute for Health Research Biomedical Research Centre funding scheme. The Dr Foster Unit at Imperial College London is largely funded by a research grant from $\mathrm{Dr}$ Foster Intelligence (an independent health service research organization) and is affiliated with the Centre for Patient Safety and Service Quality at Imperial College Healthcare National Health Service Trust, funded by the National Institute for Health Research.

Potential conflicts of interest. All authors report no conflicts of interest relevant to this article. All authors submitted the ICMJE Form for Disclosure of Potential Conflicts of Interest, and the conflicts that the editors consider relevant to this article are disclosed here.
To the Editor-Catheter-associated urinary tract infections (CAUTIs) are a complication of urinary catheterization in hospitalized patients and represent a threat to patient safety. ${ }^{1}$ CAUTI surveillance is a key tool to track the prevalence of this complication and measure the impact of prevention interventions. One key component of an effective surveillance program is valid measures of infection. Candida species are commonly isolated from the urine of hospitalized patients and may not represent urinary tract infection. ${ }^{2,3}$ We believe including Candida species as part of the National Healthcare Safety Network (NHSN) surveillance definition introduces 